\title{
Évaluation et validation des tests diagnostiques dans le cadre du ciblage thérapeutique
}

\author{
Paul Landais ${ }^{1 \star}$, Valérie Méresse ${ }^{2 \star}$, Jean-Claude Ghislain ${ }^{3}$ et les participants à la table ronde $n^{\circ} 4$ de Giens \\ $X X I V^{\star \star}$
}

1 Hôpital Necker, Service de Biostatistique, Paris, France

2 F Hoffmann-La Roche Ltd, Basel, Suisse

3 Afssaps, Saint-Denis, France

Texte reçu le 15 octobre 2008 ; accepté le 20 novembre 2008

\section{Mots clés : \\ theranostic ; \\ médicament ; \\ test diagnostic ; \\ développement ; \\ recommandations}

\begin{abstract}
Résumé - Par théranostic, on entend une combinaison particulière de l'utilisation couplée d'un médicament et d'un test diagnostique (parfois nommé « compagnon diagnostique »). Le test diagnostique désigne ici le moyen technique utilisé pour identifier un biomarqueur qui permet de moduler l'usage du nouveau médicament.

Le théranostic constitue un progrès significatif pour une médecine plus personnalisée, mieux adaptée aux patients en termes d'efficacité et de tolérance. Toutefois, le développement des théranostics est complexe car il nécessite un phasage à tous les stades, du développement au remboursement, avec une approche collaborative et maîtrisée.

Le groupe de travail a émis des recommandations comme base de réflexion et d'action pour les acteurs impliqués dans le développement de théranostics.

En effet, toutes les étapes du système répondent à des règles hétérogènes et asynchrones qu'il conviendrait d'harmoniser pour permettre une mise à disposition simultanée et un accès généralisé au médicament et au test. Des simplifications réglementaires sont nécessaires pour permettre une meilleure adaptation du théranostic à l'accélération de l'innovation.
\end{abstract}

\section{Le théranostic}

Par théranostic, on entend une combinaison particulière de l'utilisation couplée d'un médicament et d'un test diagnostique (parfois nommé « compagnon diagnostique »).

Plus précisément, le test diagnostique désigne ici le moyen technique utilisé pour identifier un biomarqueur. Selon la définition proposée par le National Institute of Health, un biomarqueur est « une caractéristique qui est objectivement mesurée et évaluée comme un indicateur de processus biologiques normaux ou pathologiques, ou de réponses pharmacologiques à une intervention thérapeutique $» .{ }^{[1]}$

Le résultat du test diagnostique permet de moduler l'usage du nouveau médicament selon trois modalités :

- sélectionner une population cible. Celle-ci sera constituée des patients dont la réponse au biomarqueur permet de présumer

\footnotetext{
* Ces auteurs ont contribué de façon analogue au travail.
}

$\star \star$ Pour la liste des participants, voir en fin d'article. que leur tolérance et/ou leur réponse au nouveau médicament sera bonne;

- déterminer une posologie individualisée du nouveau médicament,

- suivre et contrôler la réponse du patient au traitement choisi.

Le théranostic ouvre ainsi des perspectives d'une utilisation plus ciblée et individualisée des nouveaux traitements.

Le théranostic se distingue de la classique alliance médicament - test diagnostique (par exemple, le couple mesure de la glycémie - prescription d'hypoglycémiants) par au moins deux aspects :

- le développement intégré et simultané du nouveau test diagnostique et du nouveau médicament,

- une prise en compte de la réalisation couplée du test diagnostique et du nouveau médicament lors de l'attribution de l'Autorisation de Mise sur le Marché (AMM). 


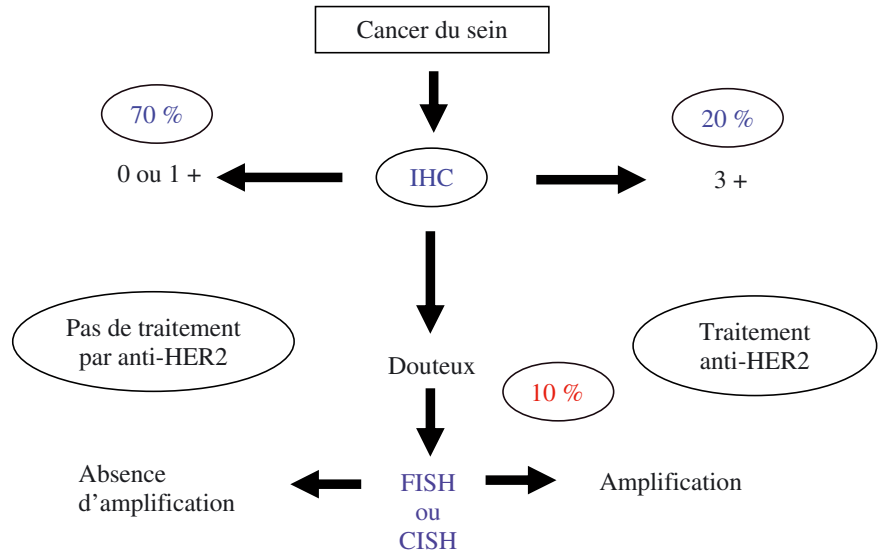

Fig. 1. Cancer du sein et stratégie d'utilisation des thérapeutiques ciblées antiHER2 en fonction du statut Her2 évalué par immuno-histochimie (IHC) et hybridation in situ (FISH). Exemple du traitement par trastuzumab en situation adjuvante.

Il existe toutefois une exception à cette dernière proposition. En effet, le développement d'un nouveau test diagnostique pour un nouveau biomarqueur pourra, dans certains cas, rester postérieur à l'attribution de l'AMM de son médicament couplé.

\section{Exemples}

Le théranostic peut être illustré par 2 exemples de «couple» médicament et test compagnon.

\section{1. Thérapeutique ciblant HER2 (trastuzumab, lapatinib)}

Her2 appartient à la famille des «Human Epidermal Receptors » (HER) et est un facteur de régulation important pour la croissance et la différenciation des cellules normales. Environ $15 \%$ des cancers du sein s'accompagnent d'une surexpression de Her2 qui s'évalue par immunohistochimie (IHC) [surexpression cotée «+ 3 »] et/ou par l'amplification du gène déterminée par hybridation in situ fluorescente (FISH) ou chromogénique (CISH) [figure 1]. Les patientes présentant un cancer du sein avec surexpression de Her2 ont un pronostic plus sombre que la population de patientes qui ne surexpriment pas le gène ; le risque de rechute est plus élevé et la survie globale est plus courte. Le trastuzumab (Herceptin ${ }^{\circledR}$ ) est un anticorps monoclonal humanisé qui se lie spécifiquement au domaine extracellulaire de la protéine Her2. Cet anticorps a été développé dans le traitement du cancer du sein métastatique et a démontré à la fois son efficacité et sa bonne tolérance dans le cadre d'essais cliniques randomisés. ${ }^{[2-4]}$ Cela a aussi été montré dans le traitement adjuvant du cancer du sein, quand le trastuzumab est administré seul ou en association avec différents régimes de chimiothérapies. ${ }^{[5]}$
Seules les patientes qui surexpriment Her2 (score +3 en IHC ou FISH/CISH positifs) sont susceptibles de bénéficier d'un traitement par trastuzumab. En cas de tumeur présentant un score +2 en IHC, une recherche complémentaire de l'amplification du gène par FISH ou CISH est nécessaire pour savoir si la patiente est éligible à un traitement par trastuzumab. Dans ces cas particuliers, cet anticorps sera prescrit uniquement si une amplification est avérée.

L'algorithme de traitement du cancer du sein, fondé sur l'emploi du trastuzumab, sera donc dépendant de la surexpression de l'oncogène Her2 évalué par IHC (ou de l'amplification de l'oncogène Her2 évalué par FISH) [ou CISH] [figure 1], qui orientera l'utilisation des thérapeutiques ciblant les récepteurs HER2 (exemple du traitement par trastuzumab en situation adjuvante) [figure 2]. La technique IHC est la méthode de dépistage la plus diffusée pour détecter une surexpression de Her2, car elle est la moins coûteuse et la seule remboursée à ce jour en France. Cependant, pour être fiable, elle doit être calibrée par rapport à la technique FISH ou CISH. En France, sous l'égide du Groupe d'Étude des Facteurs Pronostiques Immunohistochimiques dans les Cancers du Sein (GEFPICS), une étude de contrôle de qualité (le protocole «FISH 2002 ») a été conçue pour analyser la concordance IHC-FISH sur un grand nombre de tumeurs primitives du sein au stade métastatique $(\mathrm{n}=1500)$ dans des services de pathologie français. Cette étude avait pour objectif principal d'étudier la corrélation entre les techniques IHC et FISH. Elle avait aussi pour ambition de montrer l'importance du calibrage de la technique IHC sur la technique FISH. Par ailleurs, une politique d'assurance qualité est réalisée avec des tests techniques et d'interprétation développés par l'AFAQAP (Association Française pour l'Assurance Qualité en Anatomie Pathologique) et des ateliers pratiques. ${ }^{[6]}$

L'introduction récente du lapatinib est un autre exemple d'identification d'une population très ciblée (Lapatinib Procedure N. EMEA/H/C/795 Doc.Ref. : EMEA/302222/2008).

\subsection{Cetuximab et test évaluant la mutation de l'oncogène KRAS}

Le cetuximab vient d'obtenir une autorisation de mise sur le marché en première ligne du cancer colorectal en fonction du statut muté ou non du gène KRAS. Ceci est décrit dans le nouveau résumé des caractéristiques du produit (RCP) de cetuximab : «......Erbitux ${ }^{\circledR}$ est indiqué dans le traitement des patients présentant un cancer colorectal métastatique avec gène KRAS de type sauvage exprimant le récepteur du facteur de croissance épidermique (EGFR) en association avec une chimiothérapie, en monothérapie après échec d'un traitement à base d'oxaliplatine et d'irinotécan et en cas d'intolérance à l'irinotécan....... En effet, 


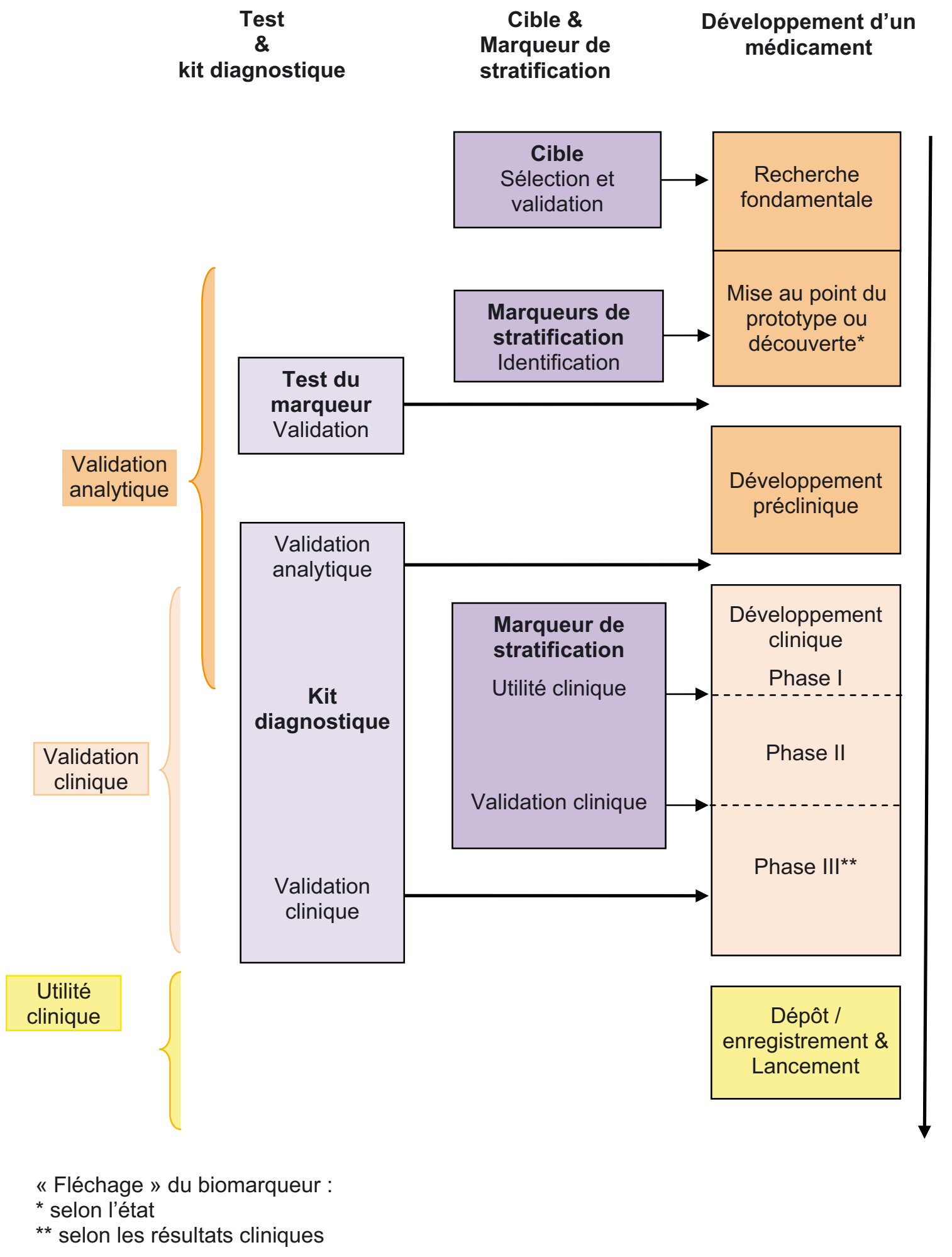

Fig. 2. Les étapes du développement d'un médicament couplé à celui d'un biomarqueur (adapté de ${ }^{[7]}$ ). 
seule la sous-population de patients ne présentant pas la mutation du gène KRAS est susceptible de bénéficier du traitement par cetuximab, la mutation conférant un risque très spécifique de résistance au traitement par cetuximab. ${ }^{[7]}$ L'identification des mutations KRAS comme marqueur de résistance aux anticorps anti-EGFR a été faite a posteriori de la commercialisation du cetuximab. ${ }^{[8]}$ Le test a été retenu dans un premier temps par les autorités européennes devant la démonstration apportée par le panitumumab avec son étude pivot qui montrait la valeur prédictive du testpour l'efficacité de ce médicament en troisième ligne en monothérapie. ${ }^{[9]}$ Ces premiers travaux ont imposé le test qui a ensuite montré son intérêt en première ligne. ${ }^{[10]}$ L'AMM du cetuximab a été modifiée secondairement pour toutes les lignes thérapeutiques. La détection des mutations de KRAS est fondée sur une réaction de PCR (Polymerase Chain Reaction) et une caractérisation des différentes mutations par séquençage, microséquençage, discrimination allélique...

Dans un article récent, Karapetis et al. ont montré l'importance de la mutation KRAS comme facteur prédictif de réponse au traitement par cetuximab. ${ }^{[1]}$ Une analyse de la mutation KRAS et sa corrélation avec la survie a été effectuée sur un échantillon de 394 tumeurs colorectales métastatiques provenant des 572 patients $(68,9 \%)$ randomisés pour recevoir un traitement par cetuximab associé aux soins de support ou des soins de support seuls. Le statut mutationnel KRAS (42,3\% des cas) était corrélé négativement à l'efficacité du traitement par cetuximab $(\mathrm{P}=0,01$ et $\mathrm{P}<$ 0,001 pour l'interaction de la mutation KRAS avec la survie globale et la survie sans progression, respectivement). Les patients ayant un statut KRAS «sauvage » bénéficiaient de façon significative du traitement par cetuximab à la fois pour la survie globale (médiane 9,5 vs. 4,8 mois ; $\mathrm{P}<0,001$ ) et la survie sans progression (médiane 3,7 mois vs. 1,9 mois ; $\mathrm{P}<0,001$ ), les patients ayant une mutation KRAS de leur tumeur ne bénéficiaient pas d'un traitement par cetuximab. La mutation KRAS ne semblait pas conférer un plus mauvais pronostic en terme de survie globale au sein du groupe de patients qui était traité par soins de support seuls. Cette publication met en exergue la nécessité d'utiliser chez tous les patients avec un cancer du colon métastatique un test diagnostique. Ce test permettra d'évaluer de façon validée et standardisée un biomarqueur (mutation KRAS) afin de sélectionner la souspopulation de patients porteurs de la mutation KRAS (42\%) qui ne bénéficiera pas d'un traitement par le cetuximab. ${ }^{[12]}$

\subsection{Adaptation posologique grâce à la mesure des concentrations sanguines du médicament (suivi thérapeutique pharmacologique)}

Un des aspects du ciblage thérapeutique est l'adaptation individuelle de la posologie. Les inhibiteurs de tyrosine kinase sont concernés puisqu'il a été montré que la réponse thérapeutique des patients traités par imatinib pour leucémie myéloïde chronique est influencée par les concentrations sanguines de ce médicament. ${ }^{[13,14]}$ De la même façon, les concentrations sanguines des anticorps thérapeutiques influencent leur efficacité, comme cela a été montré chez des patients traités par rituximab pour lymphome malin non Hodgkinien. ${ }^{[15,16]}$ Cependant, il n'existe pas encore de recommandations pour une adaptation posologique individuelle en fonction des concentrations sanguines.

\subsection{Facteurs prédictifs des effets indésirables}

Les tests diagnostiques doivent également viser à identifier les patients à risque d'effet indésirable. Une étude récente montre que les réactions d'hypersensibilité observées chez $3 \%$ des patients traités par cetuximab, un anticorps anti-EGFR, pourraient être liées à la préexistence chez certains patients d'immunoglobuline $\mathrm{E}$ (IgE) dirigés contre un sucre présent sur ce biomédicament. ${ }^{[17]}$ L'intérêt éventuel d'une recherche de ces IgE (anticorps anti- $\alpha$-gal) avant de débuter un traitement par cet anticorps monoclonal mériterait d'être étudié.

\section{Recommandations}

\section{1. Synchronisation du développement : test diagnostique et traitement}

L'intérêt du test se situe à différents étapes du développement d'un médicament comme l'illustre la figure 2.

Le constat qui est fait au sein du groupe de travail est une absence de synchronisation dans l'évaluation et la mise à disposition du couple «test diagnostic et médicament » : absence de synchronisation du développement du médicament et du test diagnostic (les procédures concernant le médicament étant généralement plus rapides); prise en charge réglementaire par des organismes différents pour la mise sur le marché et pour le remboursement par la collectivité.

Les procédures de développement du couple «médicament et test» doivent être structurées d'emblée, et validées le plus en amont possible. Nous conseillons de réaliser une double validation analytique et clinique du test diagnostique (nota bene : ces recommandations devraient pouvoir se transposer à une mesure in vivo (ex. : techniques d'imagerie fonctionnelle). Il faut favoriser les évaluations du couple (test-médicament) de façon prospective plutôt que différée. Une des difficultés du couplage d'un test et d'un médicament vient du fait qu'il peut s'agir d'un simple test mais aussi d'une combinaison de plusieurs tests (un set de biomarqueurs géniques par exemple), que ce ou ces tests peuvent être 
invasifs ou non (par exemple, biopsie ou prélèvements sanguins) et faire référence à des réglementations aujourd'hui distinctes. Par ailleurs, le développement simultané du test et du médicament caractérise généralement un processus de couplage en forme d'allers et retours de techniques et de connaissances qui traduit la complexité de la mise en œuvre.

Les procédures de qualification du biomarqueur devraient être utilisées dans ce cadre, notamment en suivant la guidance EMEA (en cours de finalisation).

Que le développement du test soit industriel ou non et selon le statut de propriété intellectuelle, un certain nombre de mesures sont recommandées :

- favoriser les bonnes pratiques de développement sur le plan analytique;

- mettre en place des recommandations de validation/qualification de la technique de mesure du biomarqueur;

- recueillir l'avis scientifique des agences réglementaires [EMEA (European Medicines Agency), Afssaps (Agence française de sécurité sanitaire des produits de santé)] sur la pertinence et la validation scientifique du biomarqueur en amont du développement du couple «test - médicament»;

- favoriser l'accès et l'exploitation de la collection d'échantillons biologiques des études cliniques, académiques et/ou industrielles ;

- et stimuler le couplage avec les données cliniques.

Un cadrage juridique est à prévoir. La table ronde $\mathrm{n}^{\circ} 7 \mathrm{de}$ la réunion des XXIV rencontres de Giens s'est penchée sur ce point.

Il est d'autre part nécessaire de favoriser des études de preuve de concept par des académiques, si possible en coordination avec les industriels, dans le but de valider de nouveaux biomarqueurs applicables à des médicaments déjà sur le marché (guichets Agence Nationale de la Recherche, Programme hospitalier de Recherche Clinique, Institut National du Cancer, INSERM, ou Direction de l'Hospitalisation et de l'Organisation des Soins).

Il faudra aussi lever les limites méthodologiques à l'évaluation de biomarqueurs sur des populations cible de petits effectifs et pour les essais post-AMM. ${ }^{[18]}$ Les efforts de développement du théranostic devraient pouvoir être valorisés en cas d'efficacité accrue ou de meilleure tolérance sur une population ciblée (par ex., via l'Amélioration du Service Médical Rendu).

Enfin, il faudrait associer les patients à la recherche des biomarqueurs, et en particulier pour l'évaluation dans des indications «niches » (comme pour les médicaments orphelins).

Le Groupe de travail propose des recommandations afin de faciliter la synchronisation à toutes les étapes de développement du couple «médicament-test diagnostic » et de permettre leur mise à disposition simultanée.

\subsection{Recommandation concernant la validation analytique du test et aspect métrologique}

Nous ne reviendrons pas sur les bonnes pratiques de développement sur le plan analytique, ni sur la mise en œuvre des recommandations de validation et de qualification de la technique de mesure du biomarqueur évoquées ci-dessus. Soulignons que dans le futur, les tests diagnostiques feront de plus en plus appel à des technologies innovantes qui évoluent en permanence, qu'elles soient fondées sur la génétique (SNP's), la transcriptomique (multiplex RT PCR), la protéomique (Elisa), ou l'imagerie.

Nous recommandons une validation et une standardisation pré-analytique de la ressource biologique et que soient appliquées les bonnes pratiques européennes ou les recommandations en cours des tests diagnostiques selon leur type : génétique, imagerie, pharmacocinétique ou anatomopathologique.

Des critères de validation pour les tests innovants devront être définis.

En cas de test diagnostique «propriétaire», sans mise sur le marché d'un kit, la publication des performances du test, équivalente à la notice des tests $\mathrm{CE}$, est recommandée.

\subsection{Processus d'autorisation conjointe du couple médicament + test}

Le groupe recommande de générer un nouveau processus réglementaire associant le couple «médicament + test » : couplage $\mathrm{AMM}$ et homologation (marquage $\mathrm{CE}$ ), et d'initier une procédure synchronisée d'accès au marché afin de permettre une mise à disposition conjointe du traitement et de son test. Une responsabilisation de tous les acteurs est nécessaire. Lors de la demande d'AMM du médicament, 3 situations sont identifiées pour le test diagnostic : soit le test industriel est déjà mis sur le marché (marquage $\mathrm{CE}$ ), soit le test industriel n'est pas encore mis sur le marché, soit il s'agit d'un test «maison» non soumis à marquage CE.

Les recommandations pour le couple «médicament - test diagnostique » sont les suivantes :

- le dossier d'AMM du médicament devrait faire mention des caractéristiques du test, de sa validité, et de la démonstration scientifique de sa pertinence;

- si le test est mis sur le marché dans l'indication de ciblage, il doit faire l'objet d'un marquage CE, être explicité dans le Résumé des Caractéristiques du Produit et faire référence au dossier clinique (dossier d'AMM);

- enfin, le cas de prestations d'analyse par un industriel concepteur du test peut se présenter. Il correspond à la mise en œuvre d'un test non mis sur le marché mais qui devrait être néanmoins conforme à la directive de marquage $\mathrm{CE}$, ou alors être assimilé à une activité de biologie médicale. 
Délai réponse HAS

$=6$ mois $(+6$ mois $)$

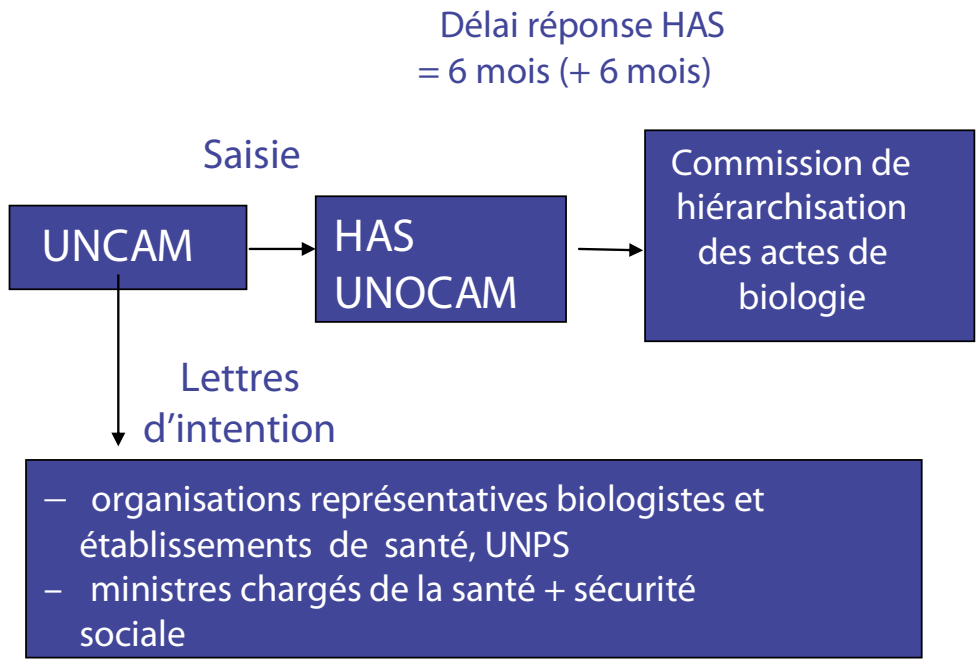

- UNCAM, HAS, UNOCAM, UNPS

- organisations représentatives

biologistes et établissements de santé

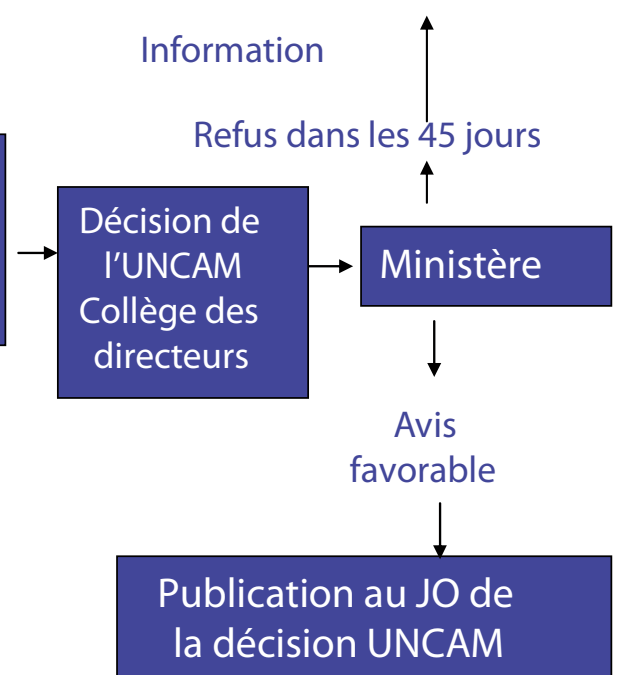

Fig. 3. Circuit d'inscription d'un acte de biologie sur la liste des actes et prestations remboursables.

UNPS : Union Nationale des Professionnels de Santé; HAS : Haute Autorité de Santé ; UNCAM : Union Nationale des Caisses d'Assurance Maladie; UNOCAM : Union Nationale des Organismes Complémentaires d'Assurance Maladie; JO : Journal Officiel.

\subsection{Recommandations pour un processus de remboursement conjoint}

Le circuit d'inscription d'un acte de biologie (ou d'anatomopathologie) sur la liste des actes et prestations remboursables est un processus complexe et long (figure 3).

Dans ce processus l'évaluation d'un nouvel acte par la Haute Autorité de santé (HAS) est une étape longue qui peut durer un an - soit 6 mois + un délai supplémentaire de 6 mois lorsque des travaux complémentaires sont nécessaires - voire davantage.

Au sein de la HAS, l'évaluation du service médical rendu du médicament est soumise à la Commission de transparence, et l'évaluation du service médical attendu du test à la Commission d'évaluation des actes professionnels. Nous recommandons un déclenchement de l'évaluation commune et simultanée par les 2 commissions lors du dépôt du dossier du médicament à la Commission de transparence afin de raccourcir les délais.

Une simplification du processus d'inscription au remboursement des actes est nécessaire pour permettre des délais comparables d'évaluation afin d'assurer une mise à disposition simultanée et un accès généralisé.

Nous préconisons une modification des textes de la sécurité sociale pour aboutir à une simplification du processus d'inscription au remboursement des actes.

Nous proposons également d'envisager un budget spécifique pour les actes innovants dans l'attente de l'inscription à la nomen- clature du test. L'article 51 de la loi de financement de la sécurité sociale pour 2009 répond à cette proposition : il introduit une possibilité de prise en charge pour des actes innovants au sein de la dotation des missions d'intérêt général et d'aide à la contractualisation (MIGAC).

\subsection{Recommandations pour la diffusion du couple « traitement et test » et assurance qualité}

Il serait important de favoriser l'information et la formation des prescripteurs et des opérateurs médico-techniques et favoriser l'harmonisation des pratiques des tests, via l'organisation d'un programme national d'assurance qualité avec les centres de références et la définition des critères de qualification des plateformes de référence et des laboratoires qui pratiqueront les tests. Pour cela une mobilisation conjointe des acteurs industriels, académiques et institutionnels est nécessaire. Les rôles respectifs restent à définir au sein de la recherche translationnelle qui établit un lien entre la recherche fondamentale et la recherche clinique (figure 4).

Il est important de favoriser l'information et la formation des prescripteurs pour que les indications de prescription du couple test/médicament soient connues des professionnels et correctement appliquées.

Lorsqu'un nouveau couple test/médicament arrive sur le marché, il faut s'assurer auparavant de l'existence préalable d'une 


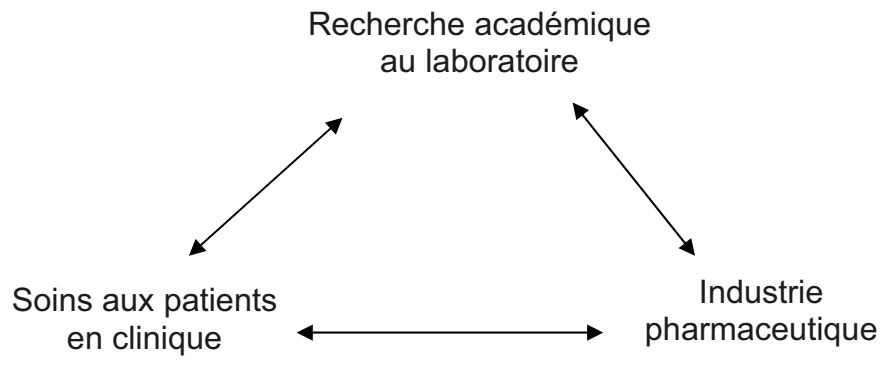

Fig. 4. Les interactions entre recherche académique, industrie et clinique.

organisation capable de réaliser les tests en respectant les conditions de qualité pour l'ensemble des patients sur le territoire. Selon le nombre de tests à réaliser par an, selon le plateau technique et les compétences spécifiques requises pour les réaliser, il peut être fait appel à des plates-formes de référence, dont il faut alors définir des critères de qualification.

Il est aussi nécessaire d'harmoniser les pratiques des tests et de préparation des échantillons, via l'organisation d'un programme national d'assurance qualité avec les centres de références ou les laboratoires qui pratiqueront les tests. La formation des opérateurs médico-techniques est aussi essentielle à ce niveau.

Une mobilisation conjointe des acteurs industriels, académiques et institutionnels est indispensable. Les rôles respectifs restent néanmoins à définir.

Enfin, il serait nécessaire de veiller au transfert de technologie entre le laboratoire utilisateur d'un test développé très en amont et sa diffusion à large échelle.

\section{Conclusion}

Le théranostic constitue un progrès significatif pour une médecine plus personnalisée, mieux adaptée aux patients en termes d'efficacité et de tolérance. Toutefois, le développement des théranostics est complexe. Il faut en particulier assurer un phasage à tous les stades, du développement au remboursement, avec une approche collaborative et maîtrisée.

Le groupe de travail a émis des recommandations comme base de réflexion et d'action pour les différents acteurs impliqués dans le développement de théranostics.

En effet, toutes les étapes du système répondent à des règles hétérogènes et asynchrones qu'il conviendrait d'harmoniser pour permettre une mise à disposition simultanée et un accès généralisé au médicament et au test.

Des simplifications réglementaires sont nécessaires pour permettre une meilleure adaptation du théranostic à l'accélération de l'innovation.
Remerciements. Samia Rahal est remerciée pour sa précieuse aide technique et Jérôme d'Enfert pour avoir aimablement accepté de contrôler le processus de mise en forme du manuscrit.

\section{Participants}

Olivier Arnaud (INSERM Tranfert), Frédéric Bibeau (CRLC Val D’Aurelle, Montpellier), Dominic Cellier (Merck Santé), Jérôme d'Enfert (Laboratoires Roche), Frédéric Eberlé (Roche Diagnostics Meylan), Agnès Goestchel (GlaxoSmithKline), Jean-Marc Grognet (Ministère de l'économie, des finances et de l'emploi), Catherine Janus (Sanofi Aventis), Behrouz Kassaï-Koupaï (EPICIM, Lyon), Pierre Laurent-Puig (Hôpital Européen Georges Pompidou, Paris), Pascale Maisonneuve (Afssaps), Frédérique Nowak (INCa), Gilles Paintaud (CHRU de Tours), Laurence Robba (CNAMTS).

\section{Références}

1. Biomarkers Definitions Working Group. Biomarkers and surrogate endpoints: preferred definitions and conceptual framework. Clin Pharmacol Ther 2001; 69(3): 89-95

2. Vogel CL, Cobleigh MA, Tripathy D, et al. Efficacy and safety of trastuzumab as a single agent in first-line treatment of HER2-overexpressing metastatic breast cancer. J Clin Oncol 2002; 20(3): 719-26

3. Slamon DJ, Leyland-Jones B, Shak S, et al. Use of chemotherapy plus a monoclonal antibody against HER2 for metastatic breast cancer that overexpresses HER2. N Engl J Med 2001; 344 (11): 783-92

4. Marty M, Cognetti F, Maraninchi D, et al. Randomized phase II trial of the efficacy and safety of trastuzumab combined with docetaxel in patients with human epidermal growth factor receptor 2-positive metastatic breast cancer administered as first-line treatment: the M77001 study group. J Clin Oncol 2005; 23 (19): 4265-74

5. Piccart-Gebhart MJ, Procter M, Leyland-Jones B, et al. Trastuzumab after adjuvant chemotherapy in HER2-positive breast cancer. N Engl J Med 2005; 353 (16): 1659-72

6. Penault-Llorca F, Balaton A, Sabourin JC, et al. Immunochemistry evaluation of HER2 status in infiltration breast cancer: technical protocol and interpretation guidelines. Ann Pathol 2002; 22 (2): 150-7

7. Phillips AK, Van Bebber S, Isssa AM. Diagnostics and biomarker development: priming the pipeline. Nature Reviews Drug Disccovery 2006; 5: 463-69

8. Lievre A, Bachet JB, Le Corre D, et al. KRAS mutation status is predictive of response to cetuximab therapy in colorectal cancer. Cancer Res 2006; 66 (8): 3992-5

9. Amado RG, Wolf M, Peeters M, et al. Wild-type KRAS is required for panitumumab efficacy in patients with metastatic colorectal cancer. J Clin Oncol 2008; 26 (10): 1626-34

10. Van Cutsem E, Lang I, D'haens G, et al. KRAS status and efficacy in the firstline treatment of patients with metastatic colorectal cancer (mCRC) treated with FOLFIRI with or without cetuximab: the CRYSTAL experience. Journal of Clinical Oncology, 2008 ASCO Annual Meeting Proceedings (Post-Meeting Edition). Vol 26, No 15S (May 20 Supplement), 2008: 2

11. Karapetis CS, Khambata-Ford S, Jonker DJ, et al. K-ras mutations and benefit from cetuximab in advanced colorectal cancer. N Engl J Med 2008; 359 (17): $1757-6$. 
12. Linardou H, Dahabreh IJ, Kanaloupiti D, et al. Assessment of somatic k-RAS mutations as a mechanism associated with resistance to EGFR-targeted agents: a systematic review and meta-analysis of studies in advanced nonsmall-cell lung cancer and metastatic colorectal cancer. Lancet Oncol 2008; 9 (10): 962-72

13. Picard S, Titier K, Etienne G, et al. Through imatinib plasma levels are associated with both cytogenetic and molecular responses to standard-dose imatinib in chronic myeloid leukemia. Blood 2007; 109 (8): 3496-9

14. Tuma RS. Disease progression in some cancers may be due to low blood levels of targeted therapies. J Natl Cancer Inst 2008; 100 (13): 912-3

15. Maloney DG, Grillo-Lopez AJ, White CA, et al. IDEC-C2B8 (rituximab) anti-CD20 monoclonal antibody therapy in patients with relapsed lowgrade non-Hodgkin's lymphoma. Blood 1997; 90 (6): 2188-95

16. Igarashi $\mathrm{T}$, Kobayashi Y, Ogura M, et al. Factors affecting toxicity, response and progression-free survival in relapsed patients with indolent
B-cell lymphoma and mantle cell lymphoma treated with rituximab: a Japanese phase II study. Ann Oncol 2002; 13 (6): 928-43

17. Chung $\mathrm{CH}$, Mirakhur $\mathrm{B}$, Chan $\mathrm{E}$, et al. Cetuximab-induced anaphylaxis and IgE specific for galactose-alpha-1,3-galactose. N Engl J Med 2008; 358 (11): 1109-17

18. Vray M, Girault D, Hoog-Labouret N, et al. Méthodologie des essais cliniques de petits effectifs. Therapie 2004; 59 (3): 273-9

Correspondance et offprints : Paul Landais, Hôpital Necker Enfants Malades, Service de Biostatistique, 149 rue de Sèvres, 75006 Paris, France.

E-mail : landais@necker.fr

Valérie Méresse, F Hoffmann-La Roche Ltd, Steinentorberg 12, 4070 Basel, Suisse. E-mail : valerie.meresse@roche.com 